\title{
RECUPERACIÓN Y ESTUDIO DE LA COLECCIÓN DE VERTEBRADOS CUATERNARIOS DEL DEPARTAMENTO DE GEOLOGÍA DE LA UNIVERSIDAD DE OVIEDO (ESPAÑA)
}

\author{
Diego J. ÁLVAREZ LAÓ \\ Universidad de Oviedo, Departamento de Geología, Área de \\ Paleontología. Arias de Velasco s/n. 33005 Oviedo (Asturias).
}

\begin{abstract}
Álvarez Laó, D.J. 2002. Recuperación y estudio de la colección de vertebrados cuaternarios del Departamento de Geología de la Universidad de Oviedo (España). [Recovery and study of the Quaternary vertebrates collection of the Department of Geology, Oviedo University (Spain).] Revista Española de Paleontologia, 17(2), $189-198$. ISSN 0213-6937.
\end{abstract}

\begin{abstract}
This study deals with the recovery and revision of an ancient collection of Quaternary vertebrates from Asturias. which was made during the filties, on the initiative of some members from the "Instituto de Geología Aplicada de Oviedo". These fossils became later on a part of the collections of the Paleontology section of the Oviedo University. In the fifties the collection was quite useful in both research and teaching. The collection turned out to be of great interest because of the large number of species represented and the extensive area (covering middle and eastern Asturias) the fossils were collected from.
\end{abstract}

Keywords: Mammals, ancient collection, Quaternary, Asturias.

\section{RESUMEN}

El presente estudio aborda el trabajo de recuperación y revisión de una antigua colección de fósiles de vertebrados cuaternarios asturianos, formada en los años 50 por iniciativa de algunos miembros del Instituto de Geología Aplicada de Oviedo, y que después pasó a formar parte de las colecciones del Área de Paleontología de la Universidad de Oviedo. Esta colección sirvió en los años 50 para obtener una aproximación al conocimiento, desde el punto de vista de la investigación y la docencia, de la fauna de macromamíferos cuaternarios de Asturias. Dicha colección resultó tener un gran interés por ser relativamente elevado el número de especies allí representadas y por ser muy diversa su procedencia, que abarca una amplia área de la zona central y oriental de Asturias.

Palabras clave: Mamíferos, colección antigua, Cuaternario, Asturias.

\section{INTRODUCCIÓN}

Los estudios sobre fauna cuaternaria en Asturias comenzaron a realizarse a principios del siglo XX de la mano de personalidades como el conde de la Vega del Sella, Obermaier, Harlé o Breuil, pero casi siempre con un carácter secundario, englobados en el estudio general de cuevas en las que el interés principal se basaba en su riqueza arqueológica. El material recogido por estos investigadores pasó a formar parte de las colecciones del antiguo "Museo de Historia Natural", creado por la Universidad de Oviedo en 1895 y situado entonces en una de las dependencias del actual "edificio histórico". Este Museo desapareció tras el incendio ocurrido en el edificio durante la revolución de 1934 (Adán, 2000).

El inicio de los estudios de Geología, promovidos y potenciados por el profesor Noel Llopis Lladó, en los años 50 , llevó a la consecución de una colección de vertebrados, fundamentalmente mamíferos del Cuaternario. Algunos de estos hallazgos fueron publicados en revistas como Speleon o Breviora Geológica Astúrica. También, con parte de este material, se había organizado una exposición didáctica permanente en las vitrinas de la Sección de Geología (situada por entonces en el edificio de la Facultad de Ciencias), la cual jugaba en aquellos días un importante papel como referencia material del patrimonio geológico de la Zona Cantábrica. 


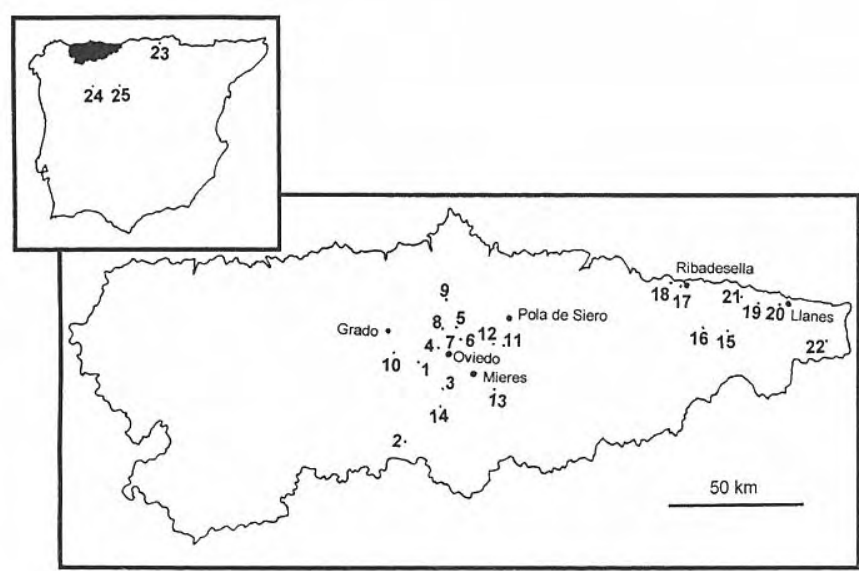

Figura 1. Localización geográfica de los yacimientos. Geographical location of the sites.

1: Tuñón-El Angel, Sto Adriano; 2: Fresnedo, Teverga; 3: La Manga del Monte, Riosa; 4: Las Caldas, Oviedo; 5: Naranco, Oviedo; 6: Tudela Veguín, Oviedo; 7: Oviedo; 8: Veneros, Oviedo; 9: San Cucao, Llanera; 10: La Xana, Grado; 11: Molledo, Siero; 12: Cierre de la Cueva, Siero; 13:Turón, Mieres; 14: Aramo, Riosa; 15: Robelledil, Onís; 16: El Milagro, Cangas de Onís; 17: La Lloseta, Ribadasella; 18: Les Pedroses, Ribadesella; 19: Balmori, Llanes; 20: El Covarón, Llanes; 21: Cueto de La Mina, Llanes; 22: Suarias, Peñamellera Baja; 23: Mañaria, Vizcaya; 24: Urdiales del Páramo, León; 25: Villamol, León.

En 1969, la Sección de Ciencias Geológicas se trasladó al edificio de la actual Facultad de Geología, lo cual hizo que desapareciera la exposición y que los materiales se quedasen provisionalmente almacenados en cajas.

Estos materiales, en virtud de su propia naturaleza y edad, habrían de quedar bastante alejados de lo que, durante casi tres décadas, constituyó el núcleo fundamental de la investigación paleontológica de la Universidad de Oviedo, es decir, el estudio de los invertebrados del Paleozoico.

La colección, no obstante, requería ser examinada, seleccionada y, en la medida de lo posible, ubicada dentro de su adecuado contexto, pues muchas de las piezas no contaban con referencias que permitieran situarlas con precisión dentro del marco geológico en el que fueron extraídas.

El estudio de esta colección trata de reabrir la línea de investigación sobre el conocimiento de los vertebrados del Cuaternario asturiano, tema que apenas había sido objeto de atención desde los años cincuenta.

La reciente puesta en marcha de una litoteca y un museo en el Departamento de Geología, supondrá nuevamente la reapertura de la exposición y, con ello, un cambio de rumbo en la conservación del patrimonio geológico y paleontológico del Departamento y de la Facultad de Geología.

\section{MATERIAL Y MÉTODOS}

\section{LA COLECCIÓN ANTES DE SU RECUPERACIÓN}

Tras la desaparición de la exposición a que hemos aludido, los materiales de la colección fueron, en su mayoría, almacenados en cajas y depositados en los almacenes del Área de Paleontología. Aunque algunas de las piezas se hallaban expuestas en una vitrina del vestíbulo de la Facultad de Geología, su emplazamiento fue cambiando con las siguientes remodelaciones del edificio, la última de las cuales tuvo lugar en 1984. Desde esas fechas, el material no había vuelto a ser consultado hasta el momento de iniciar su recuperación, en julio de 1998.

Muchas de las piezas estudiadas nunca llegaron a estar expuestas ni a ser tratadas, pues se encontraban aún en las condiciones en las que debieron de ser extraídas del yacimiento. Como paso previo a su estudio, fue necesario lavar todos los huesos para eliminar el sedimento y el polvo acumulado durante todos estos años. Además, muchos ejemplares requerían también ser reconstruidos (en los casos en que se pudieron encontrar varios fragmentos correspondientes a uno mismo) y otros muchos precisaron de su endurecido, pues su estado de deterioro y fragilidad era bastante peligroso y, de no hacerlo, quizá hubieran acabado deshaciéndose.

\section{ESTUDIO DEL MATERIAL}

Las fuentes de información fundamentales en las que me apoyé para el estudio anatómico y taxonómico del material fueron diversas.

En primer lugar utilicé, como obras de consulta, atlas de anatomía animal, tales como los de Pales y Lambert (1971), Schmidt (1972), Hue (1907) o Lavocat et al. (1966), y trabajos como los de Altuna (1972) o Torres (1984) para cuestiones más específicas, además de diversos textos. Para la cronología y encuadre prehistórico me basé en los estudios de Adán (1997) y Castaños (1992).

Fue también necesaria la formación de una colección de comparación con material actual y las visitas a las colecciones de centros tales como el Museo Geominero de Madrid, así como el contraste de opiniones con especialistas en la materia tales como los doctores Jesús Altuna, de la Sociedad de Ciencias Aranzadi de San Sebastián, o Begoña Sánchez, del Museo Nacional de Ciencias Naturales, para llegar a identificar algunas piezas y esclarecer ideas. También fue provechosa la relación con especialistas en fauna actual, como el zoólogo Carlos Nores, de la Universidad de Oviedo.

De cada ejemplar, una vez siglado, se tomaron diversas medidas y se realizó una descripción, indicando su clasificación anatómica y taxonómica, en la medida de lo posible, su procedencia (si se conoce), y algún comentario puntual, como si estaba ya clasificado o estudiado, quién lo donó y cuándo, publicaciones acerca de él, características del yacimiento, posible edad, etc.

Actualmente, las 980 piezas que componen la colección se hallan sigladas y guardadas en la Litoteca y en el Museo del Departamento de Geología de la Universidad de Oviedo. 


\section{PROCEDENCIA DEL MATERIAL Y YACIMIENTOS}

\section{FUENTES DE PROCEDENCIA}

En conjunto, esta colección fue formada principalmente en los años 50, aunque también posee aportaciones posteriores.

El material óseo es de origen muy diverso. Parte de los restos proceden de investigaciones espeleológicas, tales como los correspondientes a las cuevas de Balmori, Fresnedo, La Lloseta, etc.

La minería es otra fuente de procedencia. No era raro que en las labores mineras, desde el siglo XIX, se toparan con cuevas o cavidades cársticas en las que se hallaban restos de vertebrados. En este sentido, pueden citarse casos como el de la mina "El Milagro" de Mestas de Con (Cangas de Onís).

De la cantería se puede decir casi lo mismo. El hecho de que en Asturias se exploten gran cantidad de canteras de caliza hace de esta labor una importante fuente de aporte de estos restos. Pueden citarse casos importantes como los de Tudela Veguín, El Naranco o Las Caldas. En los casos citados, los hallazgos eran puestos a disposición del personal del Instituto de Geología Aplicada de la Universidad de Oviedo. Las prospecciones realizadas por personas como los profesores Noel Llopis, José A. Martínez o Manuel Julivert, a veces junto con investigadores paleolíticos como Francisco Jordá, dieron lugar igualmente a importantes hallazgos de este tipo. Los propios alumnos de la Sección de Ciencias Geológicas alimentaron también la colección con ejemplares que ellos aportaban como labor asignada por profesores, como Eduardo de Fraga Torrejón.

Consultando publicaciones de la época, se puede llegar a la conclusión de que sólo una parte de los restos de vertebrados conocidos en Asturias hasta los años 50 se hallaba en la colección. El resto se encontraba en el Museo Arqueológico de Oviedo, en colecciones privadas, o en otras instituciones no asturianas, como el Museo Nacional de Ciencias Naturales de Madrid o el Museo de Sabadell.

Gran parte de los restos contienen referencias acerca de su procedencia, aunque muchos otros no las tienen. Buena parte de estos últimos deben de provenir de los mismos yacimientos que los que sí están ubicados, aunque ello no conste.

En su casi totalidad, los materiales proceden de yacimientos asturianos (Fig. 1). Es importante señalar que los restos correspondientes a cada yacimiento muchas veces son poco representativos de los mismos, en contraste con los resultados obtenidos en investigaciones posteriores más profundas. También debe indicarse que la mayoría de los fósiles son huesos diagnósticos, fruto de una recogida selectiva, tal y como se solía hacer en la primera mitad del siglo $\mathrm{XX}$, en que se despreciaban los restos más pequeños y fragmentados, que muchas veces podían contener una información tafonómica, taxonómica y ambiental muy valiosa. Este tipo de muestreos podía dar lugar a conclusiones erróneas.

Los ejemplares cuya localización es conocida, proceden de los siguientes yacimientos:

\section{YACIMIENTOS ASTURIANOS}

\section{Cueva de Tuñón o del Ángel}

Próxima a la localidad de Tuñón (Santo Adriano) en el valle del río Trubia. Está formada en la Caliza de Montaña. Ya Llopis (1956) realizó una exploración de la cueva y estudió su fauna y sedimentos.

Los restos de esta localidad presentes en nuestra colección (se supone que fueron recogidos por Llopis en su exploración de la cueva) constan de unos molares de ovicáprido indeterminado y algunos molares de ciervo.

Hallazgos arqueológicos sitúan la edad de este yacimiento entre un Auriñaciense y un Aziliense Asturiense, según Adán (1997).

\section{Cuevas de Fresnedo}

Ubicadas cerca de la localidad de Fresnedo (Teverga), en el cauce del río San Pedro, a su paso por la Caliza de Montaña. Se realizaron algunas campañas de exploración (Julivert, 1952 y 1954a) de carácter principalmente hidrogeológico y geomorfológico. También se realizaron estudios arqueológicos, pues la cueva posee pinturas rupestres (Blas, 1982). En ninguno de estos estudios se mencionan restos de fauna.

En la colección estudiada hay una serie de restos de cabra montés procedentes de esta localidad, los cuales se supone que fueron recogidos y donados por Julivert durante sus campañas de exploración en los años cincuenta.

\section{Cueva de la Manga del Monte}

Ubicada en el Pico El Visu, Riosa. No hay en la bibliografía ningún dato sobre la procedencia de este material, formado por una única pieza correspondiente a un ovicáprido.

\section{Cantera de Las Caldas}

Situada cerca de la localidad de Las Caldas (Oviedo), se encuentra en el valle del arroyo de Las Caldas, en la vertiente contraria a la que contiene la conocida cueva del mismo nombre. Se trata de una cantera que dejó cavidades cársticas al descubierto. Los restos procedentes de este yacimiento fueron donados por el profesor José Antonio Martínez.

De esta localidad proceden una gran cantidad de huesos que, en su casi totalidad, corresponden a hiena de las cavernas (Crocuta crocuta spelaea), incorrectamente clasificada en su día como Hyaena. Es interesante señalar que gran parte de los restos corresponden a fragmentos de maxilares y mandíbulas de ejemplares principalmente juveniles, donde se encuentran representados diversos estadios de la dentición de la especie (Fig. 2.a-e). Es éste , junto con El Naranco y Tudela Veguín, uno de los yacimientos que más restos fósiles ha aportado a la colección.

En cuanto a la génesis del yacimiento, parece tratarse de una madriguera de estos animales, dado el alto porcentaje de restos pertenecientes a ejemplares jóvenes hallados, lo que nos puede mostrar una alta mortalidad en las etapas tempranas de la vida de los miembros de esta población. 


\section{Canteras del Naranco}

Aparecieron restos óseos en las canteras cercanas a la carretera que asciende al pico El Paisanu, en la Sierra del Naranco, Oviedo (Llopis, 1956), en las que se explotan calizas devónicas. Los materiales hallados fueron recogidos y depositados en la colección por Llopis y alumnos suyos, a mediados de los años cincuenta, tal como consta en las tarjetas que acompañaban a dichos restos.

El material está formado por una gran cantidad de huesos, casi todos ellos correspondientes a la misma especie, Rupicapra rupicapra. La presencia de una sola especie puede explicarse por la recogida selectiva de los ejemplares exclusivamente diagnósticos (Adán, 1997). Entre otros restos de la misma procedencia, se pudo identificar también el micromamífero Microtus arvalis y algún resto de un ave de tamaño tordo.

\section{Tudela Veguín}

Situada en el concejo de Oviedo, es ésta otra cantera que aportó una importante cantidad de restos a la colección. El progresivo avance del frente de la cantera puso al descubierto diversas cavidades cársticas fosilizadas en la Caliza de Montaña las cuales rápidamente eran destruidas por la explotación.

En algunas de ellas se hallaron restos de mamíferos, los cuales, según Llopis (1955b), fueron recogidos y cedidos por la dirección de la citada cantera. Entre el material actualmente reencontrado, figura gran cantidad de restos de ciervo, así como algunos restos de Equus caballus (caballo), Ursus spelaeus (oso de las cavernas) y Felis sylvestris (gato montés), Parte del material de oso fue estudiado por Crusafont y Villalta (1952). También Fraga Torrejón (1958) menciona estos hallazgos.

\section{Oviedo}

Procedentes de la ciudad de Oviedo, están depositados en la colección unos huesos de Equus (Asinus) sp. que, según consta en las etiquetas correspondientes, fueron hallados en las excavaciones de las obras de una calle.

\section{Cueva de Veneros}

Situada en Sta. Marina de Piedramuelle, Oviedo. No se han encontrado datos sobre este yacimiento en la bibliografía. El único resto procedente de este yacimiento que está depositado en la colección es un fémur de lobo, donado por el profesor José A. Martínez.

\section{Cueva de Llanera}

Situada en la localidad de San Cucao de Llanera. Se descubrió en 1942 al avanzar el frente de explotación de una cantera practicada en calizas devónicas. Fue visitada y descrita por Ríos (1954). La cueva desapareció poco después como consecuencia del propio avance de la cantera.

En su descripción, Ríos menciona el hallazgo de un gran molar, un fragmento de defensa y otros restos que corresponden a un proboscídeo. Estos últimos fueros trasladados al Instituto de Geología y estudiados por Crusafont y Villalta (1952), a quienes fueron cedidos, para su examen, por el profesor Noel Llopis Lladó. Según estos autores, que llevaron a cabo un profundo examen del material, el proboscídeo correspondería a la especie Elephas (Hesperoloxodon) antiquus mut. andrewsi. La edad que estos autores dan para el material se sitúa en el Cromeriense o interglaciar Günz-Mindel.

Procedentes de este yacimiento, se reencontraron 17 restos correspondientes al citado elefante, entre los que se encuentran tres piezas notables: un paladar, un fragmento de defensa y un molar (Fig. 2g), el cual ya fue objeto de análisis en la citada publicación de Crusafont y Villalta.

\section{La Xana}

Se encuentra esta cueva en la localidad de La Mata, Grado. Julivert (1954b), junto con un equipo de colaboradores, realizó un estudio de carácter hidrogeológico. No hay ninguna mención del hallazgo de huesos fósiles. En nuestra colección se encuentran algunos escasos restos de Cervus elaphus (ciervo) y de ovicáprido procedentes de esta localidad, los cuales se supone que fueron recolectados y depositados aquí por el profesor Julivert durante su citada exploración.

\section{Molledo}

La localidad se sitúa en la parroquia de Hevia, Siero. En una cantera de caliza de este lugar, se hallaron algunos restos que fueron donados por un particular. Los restos citados son una serie de piezas dentales de jabalí, ciervo y équido, formas todas ellas de amplia distribución estratigráfica.

\section{El Cierre de La Cueva}

Se trata de otro yacimiento procedente de una cantera de caliza, ésta situada en Santa Marina, Siero. Los restos hallados fueron donados por un particular y corresponden a unos molares de caballo, los cuales ya fueron citados por Fraga Torrejón (1958) en su catálogo.

\section{Turón}

Esta parroquia de Mieres es el único e impreciso dato de procedencia que posee un fragmento de asta de ciervo. Es ésta una zona de Asturias donde son frecuentes las labores mineras, por lo que no es extraño que se haya podido perforar algún carst con huesos fósiles en alguna de estas actividades.

\section{Aramo}

De las antiguas minas de cobre del Aramo (Riosa), proceden, casi con seguridad, unas cuernas de ciervo teñidas de verde por sales de cobre y con signos que hacen sospechar que pudieran haber sido usados como primitivos instrumentos de laboreo en minería. La cronología del yacimiento es pues holocénica y está datada en el Bronce antiguo (Blas, 1992).

\section{Cueva de Robelledil}

Localidad situada en el concejo de Onís. No se halló en la bibliografía ninguna referencia a este yacimiento. De aquí proceden una pieza de oso de las cavernas y otra de rebeco. No consta quien las donó.

\section{Mina El Milagro}

Se encuentra en las proximidades de Mestas de Con 

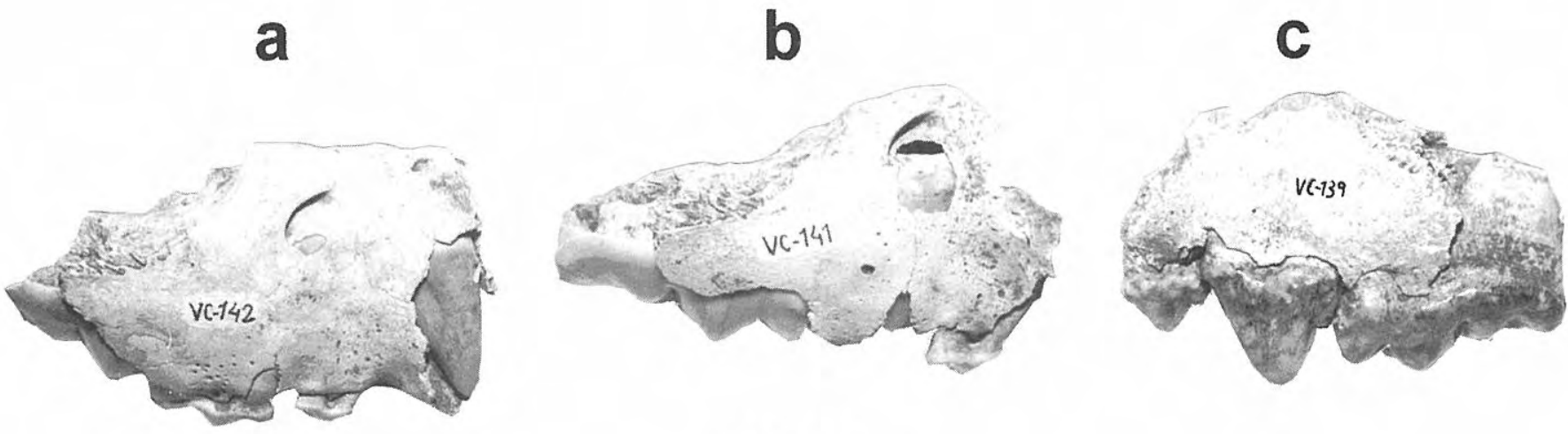

\section{$5 \mathrm{~cm}$}
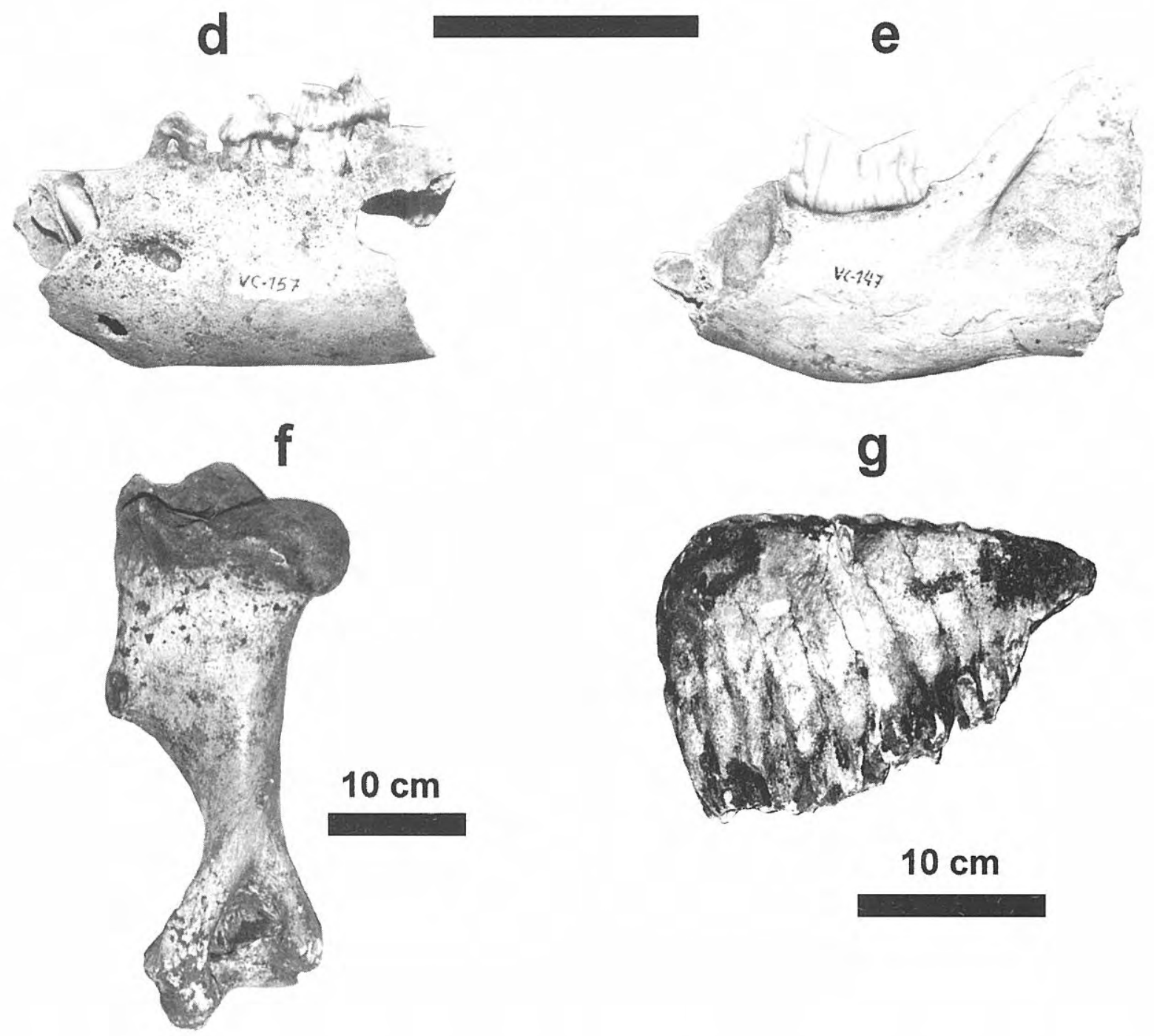

$10 \mathrm{~cm}$

Figura 2. a-e. Crocuta crocuta Erxleben: a y b, maxilares derechos de dos individuos juveniles en diferentes estadios de desarrollo (VC-142, VC-141). c, maxilar izquierdo adulto (VC-139). d, hemimandíbula izquierda juvenil (VC-157). e, hemimandíbula izquierda adulta (VC-147). (Todos en vista labial). f. Stephanorhinus etruscus Falconer: Húmero izquierdo (VC-263). Vista posterior. g. Paleoloxodon antiquus Falconer: M2 superior izquierdo (VC-737). Vista labial.

$\boldsymbol{a}$-e. Crocuta crocuta Erxleben: $\boldsymbol{a}$ and $\boldsymbol{b}$, right maxillary of two young specimens at different development stadiums (VC-142, VC-141). c, adult left maxillary (VC-139). d, young left mandible (VC-157). e, adult left mandible (VC147). (All in labial view). $f$. Stephanorhinus etruscus Falconer: Left humerus (VC-263). Posterior view. g. Paleoloxodon antiquus Falconer: Left upper M2 (VC-737). Labial view. 
(Cangas de Onís), ubicada en una cavidad cárstica de la Caliza de Montaña rellenada por arcillas cupríferas, como indica Llopis (1955a).

Esta mina ya fue explotada en el eneolítico, y se encontraron restos humanos, estudiados por Barras de Aragón (1916) y Eguren (1918), así como instrumental para el laboreo de la mina, hallazgos realizados en el siglo XIX y ya mencionados por Prado (1864), Schulz y Rada Delgado, según Fraga Torrejón (1955).

En la citada mina, en 1953, se encontró una bolsada de arcillas que contenía cobres y carbonatos de cobre (que eran objeto de la explotación) y junto con ellos, gran cantidad de huesos de mamíferos. Estos restos fueron prácticamente destruidos durante la explotación. De entre los que pudieron salvarse, algunos fueron enviados a Barcelona para su estudio por Crusafont y Villalta, y otros fueron depositados en el Instituto de Geología Aplicada de la Universidad de Oviedo. Todos estos huesos presentan la particularidad de estar más o menos impregnados por sales de cobre, debido a lo cual presentan un color verdoso que, en algunos casos, llega a ser muy intenso.

De los cuatro restos depositados en Oviedo y ya citados por Fraga Torrejón (1955), se pudieron recuperar tres, junto con algunos otros, no citados previamente, los cuales supongo que corresponderán a donaciones posteriores.

Los restos llevados a Barcelona fueron estudiados por Crusafont y Villalta, y publicados por Crusafont (1959 y 1961). La edad del depósito, según dichos autores, se sitúa en el Villafranquiense Superior debido a la presencia de la asociación de Stephanorhinus etruscus y Homotherium crenatidens.

Entre los restos de esta procedencia depositados en nuestra colección se encuentra una pieza de gran interés, un húmero izquierdo completo de Stephanorhinus estruscus (Fig. 2.f) y, junto con el, un molar perteneciente a la misma especie y algunos otros restos de ciervo, gran bóvido y un pequeño bóvido.

\section{Cueva de La Lloseta}

También conocida como cueva de La Moría o cueva de Ardines, se sitúa en la vertiente occidental del Macizo de Ardines (Ribadesella), muy próxima a la cueva de Tito Bustillo, con la que se comunica por medio de una serie de galerías (Adán, 1997).

Es ésta una cueva bastante estudiada por ser fuente de importantes hallazgos arqueológicos. Entre los restos de esta procedencia hallados en la colección se encuentra una importante cantidad de restos de ciervo y algunos de gran bóvido; también hay gran cantidad de restos de lobo y algunos de oso de las cavernas, hiena y zorro. La edad de los niveles excavados por los arqueólogos comprende desde un Magdaleniense Inferior a un Magdaleniense Medio, con un nivel Asturiense a Holoceno, según Adán (1997).

\section{Cueva de Les Pedroses}

Ubicada en las proximidades de El Carmen (Ribadesella). En esta cueva también se llevaron a cabo excavaciones arqueológicas, que nos dan unas cronologías de Solutrense Superior a un Asturiense (Adán, 1997), para los niveles estudiados.
En la colección estudiada figura un sólo resto con esta procedencia. Se trata de un metacarpiano de un bovino de reducidas dimensiones.

\section{Cueva de Balmori}

Está situada en el extremo oriental de la Sierra de La Llera, unos $600 \mathrm{~m}$ al oeste del pueblo de Balmori, en Llanes.

Es ésta una cueva muy estudiada desde principios de siglo por sus interesantes hallazgos arqueológicos. Las edades atribuidas a los niveles estudiados comprenden desde un Solutrense Superior a un Asturiense, según Adán (1997).

Los materiales contenidos en nuestra colección fueron recogidos por los profesores Llopis y Julivert, como indican las tarjetas adjuntas. Los geólogos realizaron aquí campañas de exploración (Julivert, 1953) en las que, es de suponer, recogerían dichos restos. Se trata de huesos de ciervos y de grandes bóvidos. Entre ellos se encuentran fragmentos de un cráneo, con parte de una clavija ósea de cuerno, que considero atribuible a Bos primigenius.

\section{El Covarón}

Ubicada en las proximidades de la localidad de La Pereda (Parres, Llanes). En ella se han realizado estudios arqueológicos, en los que se citan edades de Paleolítico superior, sin precisar más, y se reconoce un nivel Asturiense (Adán, 1997).

El sistema cárstico fue estudiado por Llopis (1953), y se supone que de estas campañas deben proceder los restos óseos depositados en la colección, que corresponden a ciervo, gran bóvido y lobo.

\section{Cueto de La Mina}

Se encuentra en las proximidades del pueblo de Posada de Llanes (Bricia, Llanes). Es otra de las cuevas llaniscas, tal como la de Balmori, cuya importante riqueza arqueológica dio lugar a minuciosas excavaciones y estudios. Los hallazgos óseos fueron estudiados por Castaños (1982) en niveles de edades Auriñaciense a un Asturiense, según Adán (1997).

Sólo una pieza de esta procedencia fue depositada en nuestra colección. Llopis y Jordá (1953) realizaron una visita a la cueva, siendo posiblemente ellos quienes aportaron la pieza. Se trata de un fragmento de mandíbula de caballo.

\section{Suarias}

La cueva está próxima a la citada localidad de Suarias, cercana a su vez a Panes, Peñamellera Baja. Los restos de aquí procedentes fueron recogidos y depositados en 1971 por el Dr. Miguel Arbizu, entonces estudiante, quien prospectó la cavidad. El material hallado consta de algunos restos de dentición de oso, de caballo, de ciervo, dientes y fragmentos de mandíbula de ovicáprido y bastantes restos craneales de jabalí.

\section{YACIMIENTOS NO ASTURIANOS}

\section{Cueva de Mañaria}

Este yacimiento corresponde a la provincia de Vizcaya (no se precisa más en la etiqueta que acompaña a los ejemplares). El material fue recolectado y donado por un 
alumno de la Sección de Ciencias Geológicas, en 1958. Los restos depositados corresponden a Ursus spelaeus.

\section{Urdiales del Páramo}

Este yacimiento se encuentra en las proximidades de Sta. María del Páramo, provincia de León. La etiqueta que acompaña al material (una única pieza correspondiente a ciervo), estaba firmada por Llopis. No se encontraron en la bibliografía más datos al respecto.

\section{Villamol}

Esta localidad se encuentra en las proximidades de Sahagún, León. De aquí procede un gran molar de ciervo. Tampoco se hallaron más datos con respecto a este yacimiento.

\section{RESULTADOS}

La casi totalidad de restos encontrados corresponden a macromamíferos y sólo unos pocos a micromamíferos y a aves. No se han encontrado vestigios de otros vertebrados. Los huesos de aves no fueron analizados, dada su escasez y la alta especialización que su estudio requiere. La edad de dichos restos corresponde, para la gran mayoría de los que cuentan con referencias cronológicas, al Pleistoceno Superior, habiendo algunas excepciones, como son El Milagro (Pleistoceno Inferior) y La Cueva de Llanera (Pleistoceno Medio), tal como ya se ha señalado. Las especies de mamíferos representadas en la colección son las siguientes:

\section{CARNÍVOROS}

Ursus spelaeus Rosenmüller. El oso de las cavernas es una especie muy bien representada y de amplia distribución en los yacimientos que incluye la colección investigada. Los restos de esta especie (referencias VC-1 a VC-69), corresponden a numerosos fragmentos de cráneo, piezas dentales, huesos largos, vértebras y demás partes del esqueleto postcraneal. Fueron hallados en las localidades de Robelledil, Suarias, La Lloseta y Tudela Veguín. Hay también algunos restos correspondientes a yacimientos no asturianos (Mañaria, Vizcaya). Se conocen, además, numerosas localidades asturianas donde se hallaron restos de esta especie.

Ursus arctos Linnaeus. Sólo pueden atribuirse con seguridad a esta especie un conjunto de 74 huesos (VC70 a 96) que corresponden al esqueleto postcraneal de un individuo juvenil. El material parece muy reciente. Su procedencia no consta en ninguna etiqueta. Se trata de todas las vértebras, costillas, cinturones pélvico y escapular, y algunos huesos de extremidades, que por su idéntico aspecto y grado de conservación corresponden a un mismo individuo. No hay ningún resto del esqueleto craneal.

Canis lupus Linnaeus. Está representado por numerosos restos (VC-97 a 131) de dentición, huesos largos, vértebras y demás esqueleto postcraneal procedentes de la Cueva de La Lloseta. También se encontró algún otro hueso procedente del Covarón y de Veneros.

Vulpes vulpes Linnaeus. En la colección sólo hay dos restos de zorro, correspondientes a mandíbulas (VC-132 y 133). Uno de ellos, el único cuya procedencia se conoce, fue hallado en la Cueva de La Lloseta. No obstante, es ésta una especie común en los yacimientos cuaternarios asturianos.

Crocuta crocuta Erxleben. Los restos correspondientes a hiena de las cavernas presentes en nuestra colección son muy abundantes (VC-134 a 259), haciendo de ésta una de las 3 especies mejor representadas en ella. Estos materiales proceden en su casi totalidad de un mismo yacimiento, las canteras de Las Caldas (160 restos). Constan de numerosos fragmentos de mandíbulas, maxilares, piezas dentales sueltas, huesos largos, vértebras y otros restos del esqueleto postcraneal. La mayoría de las piezas pertenecen a animales jóvenes, con dentición de leche o con doble dentición (Fig. 2.a, b y d). También, aunque escasos, hay algunos restos de dentición adulta (Fig. 2.c y e). Todo ello apunta a que en la citada caverna existía una población de estos animales, donde los individuos jóvenes serían más propensos a morir y quedar enterrados en la madriguera. Otros tres restos dentales depositados en la colección proceden de la cueva de La Lloseta.

Felis sylvestris Schreber. Una única pieza constituye la representación de esta especie en la colección (VC-260). Se trata de una mandíbula derecha procedente de Tudela Veguín. Por lo demás, esta especie no es rara en los yacimientos cuaternarios asturianos.

Panthera pardus Linnaeus. En nuestra colección hay una pieza atribuible a una extremidad de húmero de esta especie (VC-261). Su procedencia es desconocida. No obstante, se conocen más datos de presencia de leopardo en yacimientos del Pleistoceno Superior de Asturias y de la Cornisa Cantábrica.

\section{ROEDORES}

A pesar de su importancia como indicadores estratigráficos y paleoclimáticos, los restos de estos animales son prácticamente inexistentes en nuestra colección. Se supone que la causa de esto es la recogida selectiva, por parte de los recolectores, de piezas diagnósticas y de tamaño apreciable, despreciando los fragmentos o los huesos de tamaño más pequeño, pese a que pueden ser muy significativos. Los estudios de micromamíferos fósiles asturianos son muy escasos y referidos sólo a las especies de mayor tamaño, como la marmota. En nuestra colección sólo poseemos unos pocos restos correspondientes a la especie que a continuación se menciona.

Microtus arvalis Martins. En la colección hay un maxilar superior y una mandíbula de esta especie (VC-262), que procede de las canteras de El Naranco. 


\section{PERISODÁCTILOS}

Stephanorhinus etruscus Falconer. Dos piezas de esta especie, procedentes de la mina El Milagro, se encuentran depositadas en la colección (VC-263 y 264). Se trata de un gran húmero izquierdo completo (Fig. 2.f) y un molar inferior, con el color verdoso característico de su yacimiento. Que se sepa, hasta la actualidad, es éste el único yacimiento de Asturias que ha proporcionado restos de esta especie. Su edad se atribuye al Villafranquiense superior (Crusafont, 1959).

Equus caballus Linnaeus. Son numerosos los restos de caballo que poseemos en nuestra colección (VC-265 a 283). En la mayoría de los casos se trata de piezas dentales. Su procedencia es muy diversa: Cueto de La Mina, Tudela Veguín, Cierre de La Cueva, Suarias y Veneros.

Equus (Asinus) sp. Cuatro huesos de aspecto muy reciente (VC-284 a 287), atribuibles a asno, fueron hallados en unas obras en la calle González del Valle, en pleno centro del casco urbano de Oviedo, y depositados en la colección. Ha sido citado en algunos otros yacimientos cuaternarios asturianos (Fraga Torrejón, 1958).

\section{ARTIODÁCTILOS}

Sus scrofa Linnaeus. Un conjunto de restos de esta especie (VC-290 a 315) formado por fragmentos de cráneo, piezas dentales y un atlas, depositados en la colección procedentes de la localidad de Suarias. Hay también otras dos piezas dentales halladas en Molledo. No poseemos ningún otro resto del esqueleto postcraneal.

Cervus elaphus Linnaeus. Es otra de las tres especies mejor representadas de toda la colección (VC-316 a 450). Ésta incluye numerosos restos de dentición, fragmentos de cráneo, huesos largos, vértebras, otros restos del esqueleto postcraneal, y abundantes fragmentos de asta, de procedencia muy diversa. Una buena parte del material fue hallado en la cueva de La Lloseta. Otro yacimiento muy importante de esta especie es la cantera de Tudela Veguín. Hay también restos procedentes de otras localidades: cantera del Naranco, El Covarón, El Milagro, Molledo, Tuñón, la Xana, Balmori, Suarias y Turón. Otros dos restos proceden de yacimientos de Urdiales del Páramo y Villamol, ambos en León.

Es interesante la existencia de los, ya citados, tres fragmentos de asta teñidos por sales de cobre y con indicios de haber sido quizá usados como útiles prehistóricos en el laboreo de minería. Su procedencia corresponde casi con seguridad a las minas primitivas de El Aramo.

Capreolus capreolus Linnaeus. Solo poseemos una pieza atribuible a esta especie (VC-451). Se trata de un fragmento de metacarpiano cuya procedencia es desconocida.
Rupicapra rupicapra Linnaeus. Muy numerosos son los restos de rebeco depositados (VC-452 a 640), siendo la especie mejor representada de toda la colección. En su práctica totalidad, proceden de la cantera del Naranco (236 restos). Se trata de restos de dentición, fragmentos de cráneo, fragmentos de clavija ósea del cuerno, vértebras, huesos largos de todo tipo, falanges, etc., que en su conjunto representan prácticamente todo el esqueleto del animal. También hay un resto procedente de la Cueva de Robelledil. En Asturias, aún hoy es común en zonas de montaña, pero a mayores alturas que las que se hallaban en algunos yacimientos del Pleistoceno asturiano, tales como el Naranco.

Capra pyrenaica Schinz. Se ha estudiado un conjunto de restos de esta especie procedentes de la Cueva de Fresnedo (VC-641 a 686). Se trata de algunos restos de dentición, fragmentos de clavija ósea, de huesos largos y otros restos del esqueleto postcraneal.

Ovis sp. o Capra sp. En la colección hay un conjunto de restos de procedencia diversa (Cueva de Tuñón, Suarias, La Manga del Monte, Las Xanas y Las Caldas) pertenecientes al género Ovis o al género Capra (VC-687 a 703). La escasez de material, las circunstancias de su procedencia, y la ausencia de las piezas diagnósticas, impiden asignarlos con precisión a uno u otro género.

Bison priscus Bojanus. Sólo puede atribuirse con bastante seguridad a esta especie una pieza de la colección (VC-704). Se trata de una clavija ósea de cuerno de enormes dimensiones que, por su forma, sólo puede pertenecer a esta especie. No consta su procedencia. El bisonte ya ha sido citado en otros yacimientos de Asturias y de la Cornisa Cantábrica, tanto como restos óseos como en representaciones de arte parietal y mueble (Corchón, 1986).

Bos primigenius Bojanus. Los únicos restos de gran bóvido que pueden considerarse atribuibles a esta especie son un conjunto de piezas (VC-705 a 710) procedentes de la Cueva de Balmori, compuesto por varios fragmentos de cráneo, núcleo de cuerno y piezas dentales.

Bos sp. o Bison sp. Al igual que sucede con la cabra y la oveja, es facil que, sin tener los restos que podrían ser diagnósticos para diferenciar estos dos géneros, puedan llegar a confundirse, dada su similitud. Así ocurre con numerosas piezas ( $\mathrm{VC}-711$ a 735) procedentes de $\mathrm{La}$ Lloseta, Molledo, El Milagro, el Naranco, El Covarón, Les Pedroses, y otros nueve restos de procedencia desconocida. Bajo esta denominación suelen clasificarse gran parte de los restos de grandes bóvidos hallados en los yacimientos asturianos.

\section{PROBOSCÍDEOS}

Paleoloxodon antiquus Falconer. Como ya se ha indicado anteriormente, los 17 restos (VC-736 a 750c), pertenecientes a un mismo individuo, proceden de 
Llanera. Se trata de un gran fragmento de paladar con un molar, otro molar suelto (Fig. 2.g), un fragmento de defensa y otros fragmentos del esqueleto postcraneal, la mayoría de ellos difícilmente identificables. Parte de este material fue ya estudiado por diversos autores (Crusafont y Villalta, 1952; Fraga Torrejón, 1958). En Asturias se han hallado recientemente otros restos de esta especie en Buelna (Llanes) los cuales han sido estudiados por Mazo (1998) y por Pinto y Aguirre (1999). Hay también otras citas de proboscídeos no identificados, de mamut (Fraga Torrejón, 1958) y de esta misma especie en la Gruta de San Juan de Duz (Colunga) que en la actualidad se encuentran en el Museo Arqueológico de Madrid (Adán, 2000). También aparecen elefantes representados en el arte parietal y mueble de Asturias (Corchón, 1986, 1992 y 1998).

\section{AGRADECIMIENTOS}

Gracias a los doctores D. Jaime Truyols, D. Luis Sánchez de Posada y Dña. M ${ }^{\mathrm{a}}$ Luisa Martínez Chacón, por la revisión del manuscrito. Muchas gracias también a los doctores D. Miguel Arbizu, Dña. Gema Adán, D. Jesús Altuna, Dña. Begoña Sánchez y D. Carlos Nores, por su inestimable ayuda a la hora de llevar a cabo el presente trabajo.

\section{BIBLIOGRAFÍA}

Adán, G. 1997. De la caza al útil. La Industria Ósea del Tardiglaciar en Asturias. Consejería de Cultura del Principado de Asturias. Oviedo, 383 pp.

Adán, G. 2000. Los centros museísticos en Asturias durante el siglo XIX y principios del XX (1844-1934/36). AABADOM (Revista de la Asociación de Archivos, Bibliotecas y Museos del Principado de Asturias). Enero-junio-2000, Oviedo, 24-33.

Altuna, J. 1972. Fauna de Mamíferos de los Yacimientos Prehistóricos de Guipúzcoa. Munibe, San Sebastián, 24, 464 pp.

Barras de Aragón, F de las 1916. Cráneo y mandíbulas encontrados en una mina de cobre de explotación antigua en Asturias, existente en la Escuela Especial de Ingenieros de Minas. Boletín de la Real Sociedad Española de Historia Natural, 17, 331.

Blas, M.A. de 1982. La Prehistoria Reciente en Asturias. Consejería de Educación y Cultura. Oviedo, 278 pp.

Blas, M.A. de 1992. Minas prehistóricas del Aramo, Riosa. Campaña arqueológica de 1987. Excavaciones arqueológicas en Asturias- 1987-1990. Oviedo, 59-68.

Castaños, P. 1982. Estudio de Macromamíferos del yacimiento de Cueto de La Mina. Boletín del Instituto de Estudios Asturianos, Oviedo, 105/106. 43-86.

Castaños, P. 1992. Los mamíferos del Cuaternario de Eurasia. In: Paleontología de Vertebrados. Faunas y Filogenia. Aplicación y Sociedad (Ed. H. Astibia), $257-$ 286.

Corchón, M.S. 1986. El Arte Mueble Paleolítico
Cantábrico. C.I.M.A. (Centro de Investigación del Museo de Altamira). Monografía No 16. Madrid, 482 pp.

Corchón, M. S. 1992. Representación de fauna fría en el arte mueble de La Cueva de Las Caldas (Asturias, España). Significación e implicaciones en el arte parietal, Zephyrus, Universidad de Salamanca, 44-45, 35-64.

Corchón, M. S. 1998. Nuevas representaciones de antropomorfos en el Magdaleniense Medio Cantábrico. Zephyrus, Universidad de Salamanca, 51, 35-60.

Crusafont, M. 1959. El yacimiento de mamíferos del Villafranquiense Superior de "Mestas de Con" (Asturias). Speleon, Oviedo, 10 (3-4), 275-287.

Crusafont, M. 1961. El Cuaternario Español y su Fauna de Mamíferos. Ensayo de Síntesis. Speleon, Oviedo, 12 (34), 3-21.

Crusafont, M. y Villalta, J. F. 1952. Notas sobre Paleomastología del Pleistoceno en Asturias. Speleon, Oviedo, 3 (3), 93-107.

Eguren, E. del 1918. De la época eneolítica en Asturias. Boletín de la Real. Sociedad Española de Historia Natural, 17, 462.

Fraga Torrejón, E. de 1955. Nota acerca de la fauna de mamíferos fósiles de Mestas de Con. Speleon, Oviedo, 6 (4), 325-332.

Fraga Torrejón, E. de 1958. Catálogo bibliográfico de la Fauna Cuaternaria Asturiana. Monografías geológicas VIII. Instituto de Geología Aplicada, Oviedo, 3-75.

Hue, E. 1907. Musée Ostéologique. Étude de la Faune Quaternaire. Osteómétrie des mammiferees. Fasc. 1- 2. Librerie C. Reinwald, Schleicher Fréres Éditeurs, París, 186 Láms.

Julivert, M. 1952. Cuevas de Fresnedo (Asturias). Speleon, Oviedo, 3 (4), 233.

Julivert, M. 1953. Exploración de las Cuevas de Balmori. Speleon, Oviedo, 4 (3-4), 265.

Julivert, M. 1954a. Exploraciones en las Cuevas de Fresnedo. Speleon, Oviedo, 5 (1-2), 111.

Julivert, M. 1954b. Estudios hidrogeológicos en Grado. Speleon, Oviedo, 5 (1-2), 111.

Lavocat, R., Alimen, M.H., Bonifay, M.F., Bouchud, J., Brunet, M., Chaline, J., Gasc, P., Genet-Varcin, E., Prat, F. et Sauvge, J. 1966. Faune et Flores prehistóriques de L'Europe Occidentale. París, 274 pp.

Llopis, N. 1953. Estudios Hidrológicos y prehistóricos en Posada (Llanes). Speleon, Oviedo, 4 (3-4), 266.

Llopis, N. 1955a. Fauna Villafranquiense en Mestas de Con (Cangas de Onís). Speleon, Oviedo, 6 (3), 229.

Llopis, N. 1955b. Fauna cuaternaria del Karst fósil de la cantera de Tudela-Veguín (Oviedo). Speleon, Oviedo, 6 (3), 227.

Llopis, N. 1956. La fauna y los sedimentos de la Cueva de Tuñón (Asturias). Speleon, Oviedo, 7 (1-4), 145-154.

Llopis, N. y Jordá, F. 1953. Visita a Cueto de La Mina. Speleon, Oviedo, 4 (2), 105.

Mazo, A. 1998. El elefante fósil de Buelna (Asturias). Nota preliminar. Estudios Geológicos, 54 (5-6), 273-274.

Pales, L. et Lambert, Ch. 1971. Atlas ósteologique des mammiferes 1. Les membres. Editions du Centre National de la Recherche Scientifique, 132 Láms. 
Pinto, A. y Aguirre, E. 1999. Presencia del elefante antiguo Elephas (Paleoloxodon) antiquus en la Cueva de La Silluca (Buelna, Asturias). Excavaciones arqueológicas en Asturias 1995-98. Oviedo, 225-232.

Prado, C. de 1864. Descripción física y geológica de la provincia de Madrid. Imprenta Nacional. Madrid, 219 pp.

Ríos, J. M. 1954. Visita a la Cueva de Llanera. Speleon,
Oviedo, 5 (3), 225-232.

Schmidt, E. 1972. Atlas of Animal Bones for Prehistorians, Archaeologist and Quaternary Geologist. Elsevier. Londres, $243 \mathrm{pp}$.

Torres, T. de 1984. Ursidos del Pleistoceno Holoceno de la Península Ibérica. Tesis doctoral. Madrid (inédita), 653 pp.

Manuscrito recibido: 24 de mayo, 2001

Manuscrito aceptado: 22 de abril, 2002 